\title{
Línea base del mercurio, departamento de Antioquia: sedimentos activos finos de corriente escala 1:500.000*
}

\author{
Luis Hernán Sánchez Arredondo* \\ Jorge Martín Molina Escobar ${ }^{* * *}$ \\ Rafael Ignacio Machado Serrano ${ }^{* * * *}$
}

\author{
Recibido: 03/15/2017 - Aceptado: 01/11/2018 \\ https://doi.org/10.22395/rium.v18n34a1
}

\begin{abstract}
Resumen
Con base en muestras de sedimentos activos finos de corriente y su procesamiento, fue elaborada la línea base del mercurio (Hg) del departamento de Antioquia. Las muestras fueron analizadas con espectrografía de fluorescencia atómica (AFS), con un límite de detección de $2 \mu \mathrm{g} \mathrm{kg}^{-1}$. Para el modelamiento espacial se usó un grid de $100 \mathrm{~m} \times 100 \mathrm{~m}$, esto quiere decir que se estimaron en el departamento de Antioquia 18,9 millones de celdas de $10.000 \mathrm{~m}^{2}$. La cartografía se elaboró aplicando la técnica geoestadística del kriging lognormal. Los valores altos de $\mathrm{Hg}(>182 \mu \mathrm{g}$ $\mathrm{kg}^{-1}$ ) se encuentran en el nordeste antioqueño relacionados con depósitos de oro y plata, principalmente con las mineralizaciones filonianas de Segovia-Remedios, de Anorí y Bajo Cauca, donde se detectaron valores por encima de $860 \mu \mathrm{g} \mathrm{kg}{ }^{-1}$. Estos valores son muy superiores al límite permisible máximo de $150 \mu \mathrm{g} \mathrm{kg}^{-1}$, lo que evidencia la necesidad de mayor cuidado en el manejo ambiental. indicando mayor cuidado en el manejo ambiental. Otras regiones muestran valores menores. El mapa se constituye en un elemento fundamental en la planificación y manejo ambiental del departamento de Antioquia, Colombia.
\end{abstract}

Palabras clave: mercurio; kriging lognormal; Antioquia.

Este artículo es parte de una investigación cuyo objetivo fue tener el mapa geoquímico de Antioquia con base en sedimentos activos. Fue financiado con recursos de la Universidad Nacional y de la Gobernación de Antioquia.

** $\quad$ M.Sc. en Ciencia y tecnología del carbón. Profesor del Departamento de Materiales y Minerales Universidad Nacional de Colombia, Medellín, Colombia. Correo electrónico: lhsanche@unal.edu.co. Orcid: http://orcid. org/0000-0009-1501-8345

*** Ph.D. en ordenamiento territorial. Profesor del Departamento de Geociencias y Medio Ambiente Universidad Nacional de Colombia, Bogotá, Colombia. Correo electrónico: jmmolina@unal.edu.co. Orcid: http://orcid. org/0000-0002-8455-1509

**** Ph.D. en recursos naturales y medio ambiente. Profesor del Departamento de Materiales y Minerales Universidad Nacional de Colombia, Bogotá, Colombia. Correo electrónico: rimachad@unal.edu.co. Orcid: http://orcid. org/0000-0002-6068-2571 


\title{
Mercury baseline, department of Antioquia: current fine active sediments in a scale of 1:500.000
}

\begin{abstract}
Based on samples of current fine active sediments and its processing was made the baseline for mercury at the Department of Antioquia (Hg). The samples were analyzed with atomic fluorescence spectroscopy (AFS) with a detection limit of $2 \mu \mathrm{g} \mathrm{kg}^{-1}$. For space modeling, a grid of $100 \mathrm{~m} \times 100 \mathrm{~m}$ was used, so 18,9 million grids of $10.000 \mathrm{~m}^{2}$ were estimated. The cartography was made applying the kriging lognormal geo statistical technique. The high $\mathrm{Hg}$ values $(>182 \mu \mathrm{g}$ $\mathrm{kg}-1$ ) were found in the northeast of Antioquia and are related to gold and silver deposits, mainly with sub-volcanic (hypabyssal) rocks at Segovia-Remedios, Anorí and the Lower Cauca, were values higher to $860 \mu \mathrm{g} \mathrm{kg}^{-1}$ were found. These values are higher than the maximum allowed limit of $150 \mu \mathrm{g} \mathrm{kg}^{-1}$, which points out the need for better environmental management. Other regions show smaller values. So, the map becomes a fundamental part of the planning and environmental management of the Department of Antioquia, Colombia.
\end{abstract}

Keywords: mercury; kriging lognormal; Antioquia.

\section{Linha base do mercúrio, estado de Antioquia: sedimentos ativos finos de corrente escala 1:500.000}

\section{Resumo}

Com base em amostras de sedimentos ativos finos de corrente e seu processamento, foi elaborada a linha base do mercúrio (Hg) do estado de Antioquia, Colômbia. As amostras foram analisadas com espectrografia de fluorescência atômica (AFS), com um limite de detecção de $2 \mu \mathrm{g} \mathrm{kg}^{-1}$. Para o modelamento espacial, foi usado um grid de $100 \mathrm{~m}$ x $100 \mathrm{~m}$; isso quer dizer que foram estimados no estado de Antioquia 18,9 milhões de celas de $10.000 \mathrm{~m}^{2}$. A cartografia foi elaborada aplicando a técnica geoestatística do kriging lognormal. Os valores altos de $\mathrm{Hg}\left(>182 \mu \mathrm{g} \mathrm{kg}^{-1}\right)$ se encontram no nordeste de Antioquia relacionados com depósitos de ouro e prata, principalmente com as mineralizações de veias de Segovia-Remedios, de Anorí e Bajo Cauca, em que foram detectados valores por cima de $860 \mu \mathrm{g} \mathrm{kg}^{-1}$. Esses valores são muito superiores ao limite permissível máximo de $150 \mu \mathrm{g} \mathrm{kg}^{-1}$, o que evidencia a necessidade de maior cuidado no manejo ambiental, indicando maior cuidado no manejo ambiental. Outras regiões mostram valores menores. O mapa é constituído em um elemento fundamental no planejamento e no manejo ambiental do estado de Antioquia, Colômbia.

Palavras-chave: mercúrio; kriging lognormal; Antioquia. 


\section{INTRODUCCIÓN}

El mercurio (Hg) es un metal pesado de grupo II B de la tabla periódica, junto con el Zn y el $\mathrm{Cd}$. El oro y el $\mathrm{Tl}$ son sus vecinos del período. El elemento tiene un número atómico de 80, una masa atómica de 201, dos estados principales de oxidación $(+1$ y +2) y siete isótopos estables en estado natural (196 Hg, 198 Hg, 199 Hg, 200 Hg, 201 Hg, 202 Hg y 204 Hg) del cual 202 Hg es el más abundante en un 29,6 \% de la masa total, seguido por el $200 \mathrm{Hg}$ (23,3 \%) y 199 Hg (17,0 \%). El mercurio tiene una química muy diferente a la de los demás elementos de su grupo y a la de sus vecinos. Es el único metal en estado líquido a temperatura ambiente.

El Hg es un elemento calcófido muy raro, las fumarolas calientes volcánicas y las rocas sedimentarias alteradas, por la actividad freática, son las fuentes principales de mineralización del Hg. El principal mineral es el cinabrio HgS, junto con el grupo de minerales del metacinabrio en el que cantidades variables de $\mathrm{Zn}$ y Fe son sustituidas por $\mathrm{Hg}$ y Se sustituido por S. Otros minerales de $\mathrm{Hg}$ primarios, incluyen el mercurio nativo, corderoita $\mathrm{Hg}_{3} \mathrm{~S}_{2} \mathrm{Cl}_{2}$ y livingstonita $\mathrm{HgSb}_{4} \mathrm{~S}_{8}$. Los minerales de $\mathrm{Hg}$ secundarios incluyen la schuetteita $\mathrm{Hg}_{3}\left(\mathrm{SO}_{4}\right) \mathrm{O}_{2}$ y el calomel $\mathrm{Hg}_{2} \mathrm{Cl}_{2}$. El mercurio también es un rastreador de algunos sulfuros como la tetrahedrita $\mathrm{Cu}_{3} \mathrm{SbS}_{3.25}$ y la esfalerita ZnS.

El gran tamaño del ion de $\mathrm{Hg}^{2+}$ nominal, junto con su bajo grado de carácter iónico, evita su incorporación en muchos minerales formadores de silicatos. Las rocas ígneas basálticas y gabroicas, generalmente tienen mucho más bajo contenido de $\mathrm{Hg}$ (típicamente $10 \mu \mathrm{g} \mathrm{kg}^{-1}$ ) que el granito (el promedio $80 \mu \mathrm{g} \mathrm{kg}^{-1}$ ). Niveles más altos de $\mathrm{Hg}$ (por encima de $1.500 \mu \mathrm{g} \mathrm{kg}^{-1}$ [1]) se han encontrado en eclogitas y peridotitas en inclusiones en tubos (pipes) de kimberlita, sugiriendo que la corteza profunda y el manto superior pueden considerarse relativamente enriquecidos en Hg, en comparación con las rocas de la corteza superior. En rocas sedimentarias, los niveles promedios de Hg son más altos en lutitas (400 $\left.\mu \mathrm{g} \mathrm{kg}^{-1}\right)$ que en calizas $\left(40 \mu \mathrm{g} \mathrm{kg}^{-1}\right)$ y arenisca (30 $\mu \mathrm{g} \mathrm{kg}^{-1}$ ) [2]. El enriquecimiento relativo de $\mathrm{Hg}$ es más marcado en lutitas carbonosas que pueden contener varios $\mathrm{mg} \mathrm{kg}^{-1} \mathrm{Hg}$ dentro de los minerales arcillosos, en residuos orgánicos y sulfuros. Los carbones muestran niveles muy variables de Hg, de $<10 \mu g$ $\mathrm{kg}^{-1} \mathrm{a}>1000 \mu \mathrm{g} \mathrm{kg}^{-1}$, algunos niveles muy altos, de $30 \mathrm{mg} \mathrm{kg}^{-1}$, han sido reportados en petróleo crudo. Su contenido promedio en rocas de la corteza es probablemente inferior a $80 \mu \mathrm{g} \mathrm{kg}^{-1}[3]$.

El mercurio es usado en exploración geoquímica como elemento guía (pathfinder), principalmente para prospectar $\mathrm{Au}, \mathrm{Ag}, \mathrm{Cu}, \mathrm{Sb}$ y mineralizaciones de sulfuros masivos. Altos niveles de $\mathrm{Hg}$ se encuentran en rocas y suelos cerca de zonas de fallas geológicas. 
La presencia de mercurio en suelos es altamente dependiente de varios parámetros, como la concentración inicial en la solución del suelo, la presencia de otros iones (como $\mathrm{Cl}$ ), formación compleja orgánica y precipitación. El contenido de un exceso de iones $\mathrm{Cl}^{-}$parece disminuir la absorción de $\mathrm{Hg}^{+}$dentro de partículas minerales, incluyendo óxidos de Mn y otros materiales orgánicos debido a que los complejos muy estables de Hg-Cl son pobremente absorbidos [1]. La absorción del Hg por minerales arcillosos en suelos parece estar muy limitada y puede variar ligeramente con el $\mathrm{pH}$, donde la más alta absorción se da para valores de pH entre 4 y 5. Sin embargo, en suelos ácidos, puede ocurrir la formación de HgS e incluso de Hg metálico. La acumulación de Hg en suelos, por consiguiente, se controla por la presencia de complejos orgánicos y agua lluvia. Así, la movilidad del Hg requiere procesos de disolución, como la degradación biológica y química de compuestos organometálicos [1]. La transformación de compuestos de órgano-mercurio, sobre todo la metilación de Hg elemental, juega el papel más importante para el ciclo del $\mathrm{Hg}$ en el ambiente. Los suelos normales contienen típicamente entre 20 y $150 \mu \mathrm{g} \mathrm{kg}^{-1} \mathrm{Hg}$ [1] y los valores en suelos no tienden a exceder $400 \mu \mathrm{g} \mathrm{kg}^{-1}$ [4]. La acumulación de Hg está principalmente relacionada con los niveles de carbón orgánico (CO) y azufre (S) en suelos, donde las concentraciones altas ocurren en los horizontes más superficiales. El horizonte orgánico tiene un contenido más alto de Hg que el horizonte mineral, esto es debido a la alta capacidad de absorción de la materia orgánica.

En ambientes ácidos, el Hg está limitado al contenido de materia orgánica, desde donde puede lixiviarse como compuestos orgánicos, considerando que en el ambiente neutral y alcalino se lixivia como una fracción activa inorgánica. La cantidad de Hg movilizado decrece a $\mathrm{pH}<3$ y a $\mathrm{pH}>12$, debido a la extremadamente alta capacidad buffer de humos en ambos ambientes [1].

El mercurio se presenta en el agua natural en varias formas que incluyen $\mathrm{Hg}$ elemental ( $\mathrm{Hg} 0$ ) que es raro en las aguas no contaminadas, el $\mathrm{Hg}$ iónico $\left(\mathrm{Hg}^{+1} \mathrm{y} \mathrm{Hg}^{2+}\right.$ y $\mathrm{Hg}$ metilado $\left(\mathrm{CH}_{3} \mathrm{Hg}+, \mathrm{CH}_{3} \mathrm{Hg}\right)$. Existe evidencia creciente de que los materiales orgánicos naturales forman complejos fuertes con el mercurio, vía el grupo funcional sulfídico, que influye en la concentración, especiación y subsecuente bioaccesibilidad del mercurio en el ambiente acuático [5]. Los niveles promedios de Hg en el agua superficial normalmente son muy bajos, típicamente $<0.1 \mu \mathrm{g} 1-1$. Minerales de mercurio, como el cinabrio y el metacinabrio, son insolubles bajo condiciones normales y el mercurio metálico no reaccionará directamente con el agua superficial [6]. Sin embargo, este ambiente aparentemente inerte, puede ser preocupante, ya que los derrames de mercurio metálico pueden persistir dentro de sedimentos activos de corriente durante muchos años y el $\mathrm{Hg}$ puede movilizarse en cantidades pequeñas, pero significantes, por la metilación microbiana bajo condiciones favorables, lo cual está por estudiarse. 


\section{1. ÁREA DE ESTUDIO}

El estudio se realizó en el departamento de Antioquia (figura 1) entre norte $05^{\circ} 26^{\prime} 20^{\prime \prime}$ y $08^{\circ} 52^{\prime} 23^{\prime \prime}$ latitud y este $73^{\circ} 53^{\prime} 11^{\prime \prime}$ y $77^{\circ} 07^{\prime} 16^{\prime \prime}$ longitud, que comprende una extensión territorial de $63.612 \mathrm{Km}^{2}$. El departamento tiene una línea costera con la región caribe en la parte norte, y se encuentra con las cordilleras central y occidental.

El territorio antioqueño es principalmente montañoso (85 \%) con relación a las cordilleras Central y Occidental. Las tierras bajas corresponden a los valles de los ríos Atrato, Porce, Cauca y Magdalena. Las unidades morfológicas son: la cordillera Occidental, la cordillera Central, el valle del río Atrato y la región de Urabá.

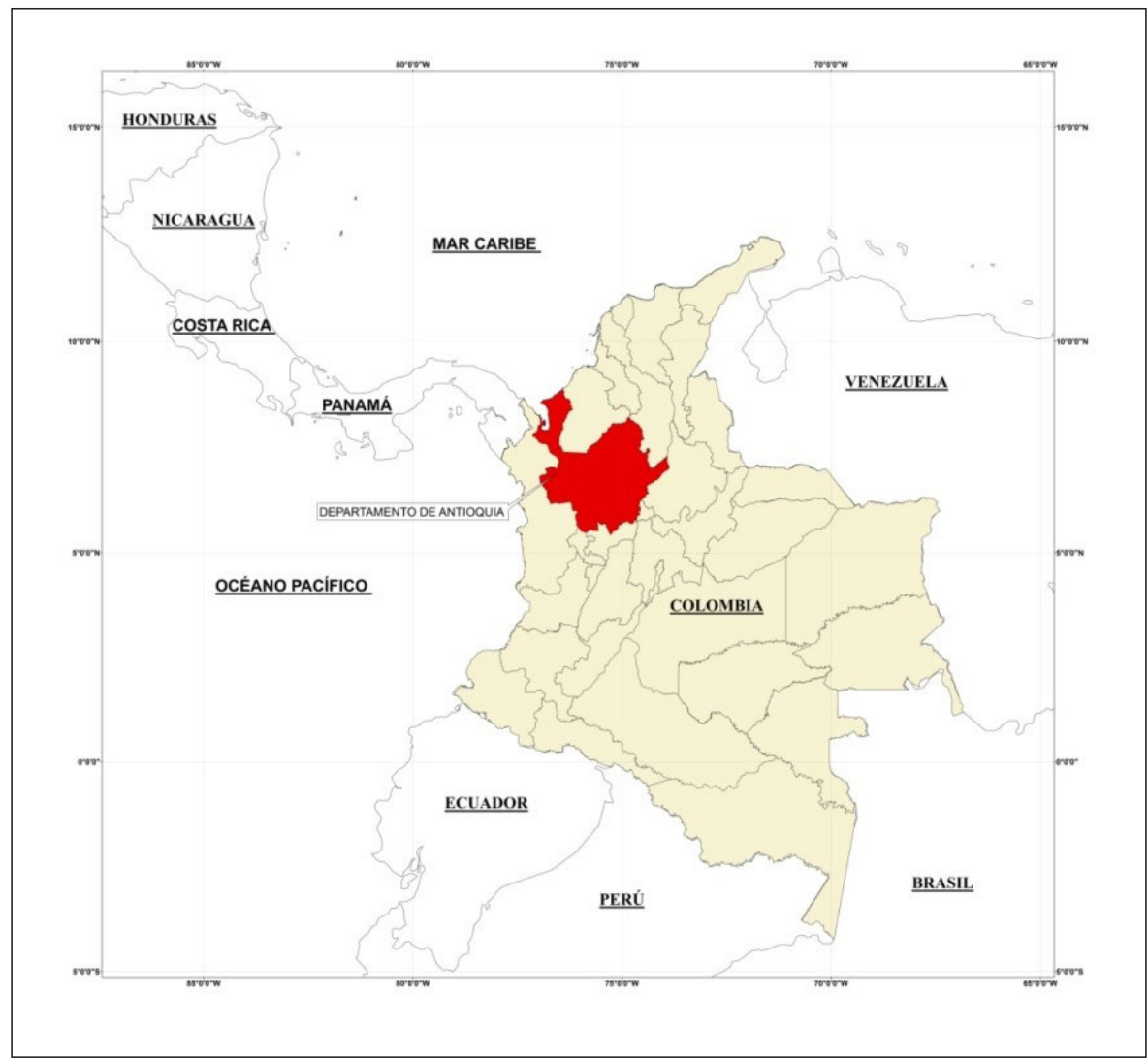

Figura 1. Ubicación del departamento de Antioquia

Fuente: elaboración propia.

El sistema de drenaje del departamento es muy extenso y está conformado por los ríos Magdalena, Cauca y Atrato que cuentan con un alto número de afluentes que cruzan el departamento entero. Las zonas termales están distribuidas en cálido (35.550 $\left.\mathrm{km}^{2}\right)$, templado $\left(16.430 \mathrm{~km}^{2}\right)$ y frío $\left(10.900 \mathrm{~km}^{2}\right)$. 
El suelo está clasificado como suelos de planicie aluvial -localizados en los ríos planos y en la costa caribe- y suelos de colinas, que son desarrollados de rocas ígneas y sedimentarias en un clima húmedo, de cenizas volcánicas y otros cimientos ígneos y metamórficos.

\subsection{GEOLOGÍA DE LA ZONA DE ESTUDIO}

En el departamento de Antioquia los afloramientos rocosos estas relacionados a litologías ígneas, metamórficas y sedimentarias, que varían en edad desde el Precámbrico al Holoceno, porque han sido agrupadas por varios autores en diferentes territorios geológicos (figura 2). Las rocas del Precámbrico al Paleozoico dominan principalmente en la cordillera Central, con la presencia de rocas metamórficas. Las unidades vulcano sedimentarias de la edad del Mesozoico principalmente afloran en la cordillera Occidental y pocos afloramientos en la cordillera Central. Varias secuencias sedimentarias típicas del sistema montañoso afloran en el centro del departamento como un romboedro con bordes largos, con mantos de carbón del Cenozoico que afloran en el golfo de Urabá, en el bajo Cauca y en el Magdalena medio.

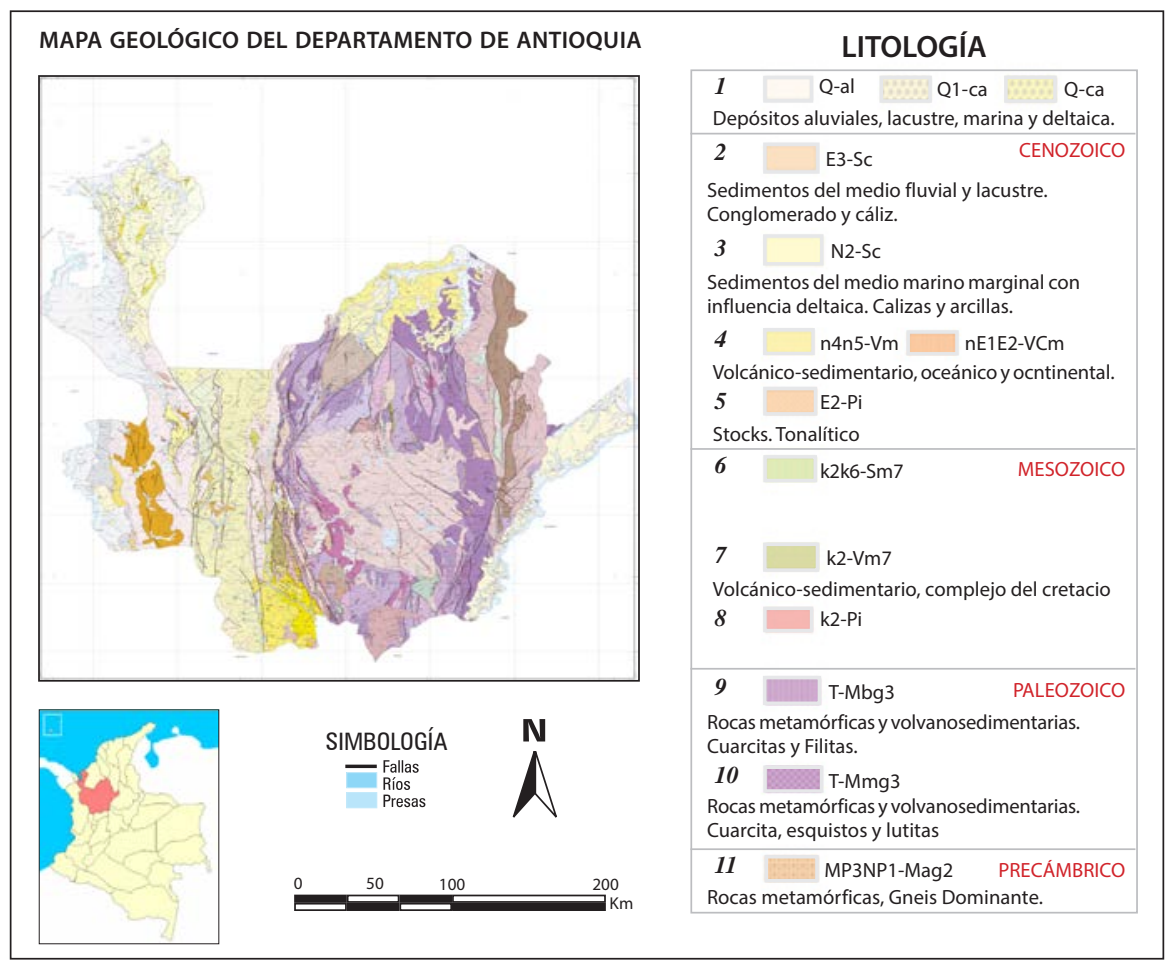

Figura 2. mapa geológico del departamento de Antioquia

Fuente: modificada de [7]. 
El departamento de Antioquia está afectado por dos sistemas de fallas regionales: el sistema de falla Palestina (PS) hacia el este y el sistema Cauca-Romeral (CRS) hacia el oeste del departamento. Ambos sistemas forman un inmenso rombo, en el que el Batolito antioqueño en más de $7.221 \mathrm{Km}^{2}$ aflora en el área central del departamento. Las direcciones de desplazamiento de estos sistemas de fallas son movimientos muy evidentes, que alcanzan cientos de kilómetros de extensión. Las principales fallas del PS son Nus, Bagre, Otú, Jetudó, Cocorná sur, Mulato y Cimitarra. El CRS es principalmente representado por las fallas Romeral, Amagá, Piedecuesta, San Jerónimo y Espíritu Santo. La falla Murindó pertenece a la región Atrato.

\section{METODOLOGÍA}

El campo de trabajo fue diseñado en un muestreo de baja densidad. Paneles de $600 \mathrm{~km}^{2}$ (30 km x $20 \mathrm{~km}$ ) fueron definidos como unidades básicas de muestreo que interceptan el departamento de Antioquia en un número de 152 celdas o paneles (figura 3). La malla de panel fue hecha con la intercepción del mapa de la división política y administrativa con escala 1:500.000, todo basado en el levantamiento geográfico de Colombia, IGAC.

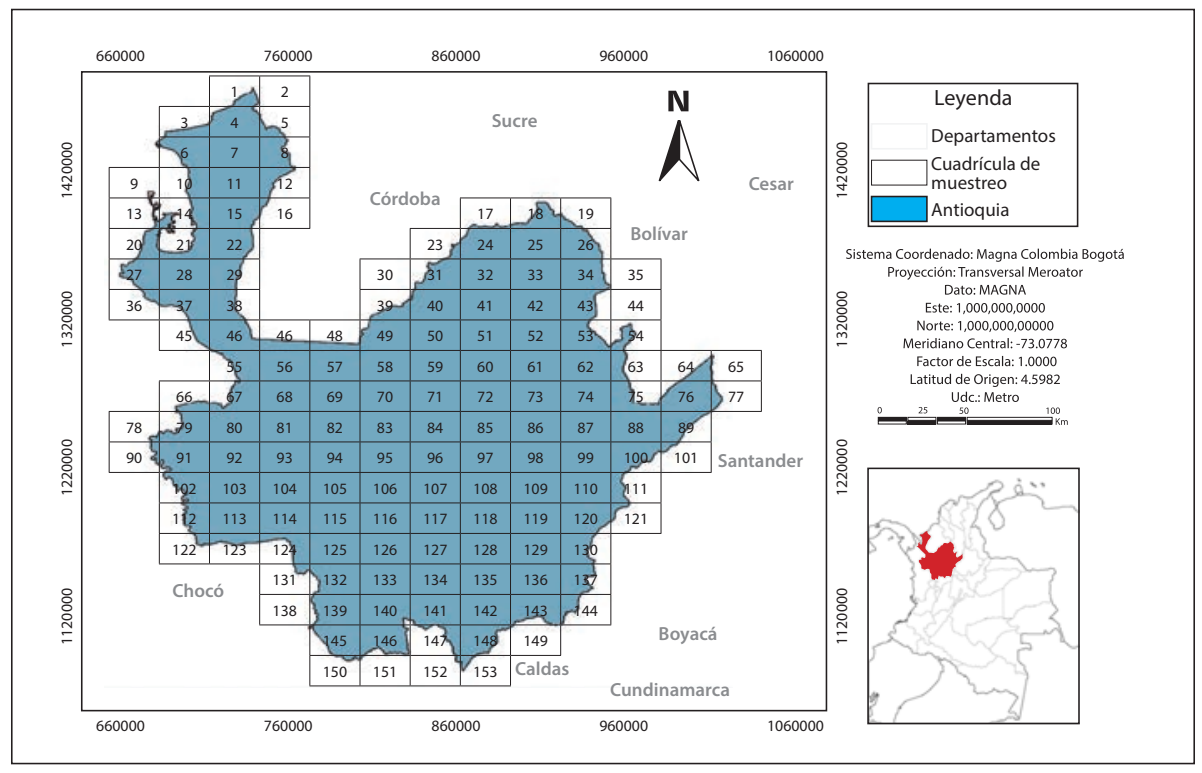

Figura 3. conformación de compositos en paneles de 600 km² [8] Fuente: elaboración propia.

Muestras de los sedimentos activos finos de corrientes fueron tomadas para obtener una composición geoquímica promedio de la cuenta de drenaje. En total 464 muestras 
fueron recolectadas, lo cual sirve como base para conformar compositos representativos de 98 paneles, cada composito representa un área equivalente de $600 \mathrm{~km}^{2}$. Las muestras fueron tomadas con herramientas y material plástico (palas, biombo, recipiente y batea). Las herramientas de muestreo siempre fueron lavadas con agua de los arroyos muestreados, antes y después de cada recolección de la muestra. Dos kilogramos de sedimentos por arroyo fueron recolectados en cada sitio de muestreo para garantizar la cantidad necesaria de material fino requerido por análisis.

\subsection{Preparación de las muestras}

Se hizo una verificación de códigos y se dispuso cada muestra en un recipiente de material no contaminado (de plástico), la limpieza se hizo manualmente para eliminar materiales, como raíces y hojas. El secado de las muestras se llevó a cabo a temperatura ambiente $\left(<40^{\circ} \mathrm{C}\right)$. Las muestras fueron tamizadas utilizando un tamiz de nailon (libre de contaminación) de $180 \mu \mathrm{m}(0,18 \mathrm{~mm})$. De acuerdo con las especificaciones de ASTM 11-70, que corresponde a la malla 80 en la designación Tyler. Por último, se hizo una mezcla homogénea para obtener $50 \mathrm{~g}$ de sedimentos. La muestra (50 g) fue envasada en recipientes de plástico y marcada con un código del panel diseñado.

Se hizo recuperación de testigos de muestras, que son producto de la fracción no utilizada. Con un tamaño de malla 80. Las muestras fueron enviadas en cajas para análisis químico en la república de China.

\subsection{Análisis geoquímico}

El análisis geoquímico fue hecho con espectrometría de fluorescencia atómica (AFS), por el Instituto de Exploración Geoquímica y Geofísica de Beijing, República de China. Los datos reportados tienen un límite de detección de dos partes por billón $\left(2 \mu \mathrm{g} \mathrm{kg}^{-1}\right)$.

\section{ANÁLISIS DE DATOS}

El análisis de los datos se hizo con estadística descriptiva, se encontró que la mediana del contenido de $\mathrm{Hg}$ en los sedimentos activos finos de corriente es $67 \mu \mathrm{g} \mathrm{kg}^{-1}$, con un rango variable entre 11 y $3.888 \mu \mathrm{g} \mathrm{kg}^{-1}$. También se definieron los percentiles (ver tabla 1).

Los datos fueron transformados al logaritmo natural para hacer un análisis variográfico. Se calcularon semivariogramas para determinar la continuidad espacial, luego se hizo una validación utilizando la técnica de la validación cruzada. Para el modelamiento espacial se usó el modelo y un grid de $100 \mathrm{~m}$ x $100 \mathrm{~m}$; el grid intercepta el departamento de Antioquia en 18,9 millones de celdas de $10.000 \mathrm{~m}^{2}$. Aplicando la técnica geoestadística Lognormal Kriging of Blocks fueron plasmados en forma espacializada estos resultados en la figura 4. 
Tabla 1: distribución del $\mathrm{Hg}$

\begin{tabular}{cc}
\hline \multicolumn{2}{c}{$\mathrm{Hg}$} \\
\hline Percentil (\%) & Concentración $\left(\mu \mathrm{kg}^{-1}\right)$ \\
\hline 5 & 34 \\
\hline 25 & 48 \\
\hline 50 & 67 \\
\hline 75 & 98 \\
\hline 90 & 182 \\
\hline 98 & 860 \\
\hline \multicolumn{2}{c}{ Fuente: elaboración propia. }
\end{tabular}

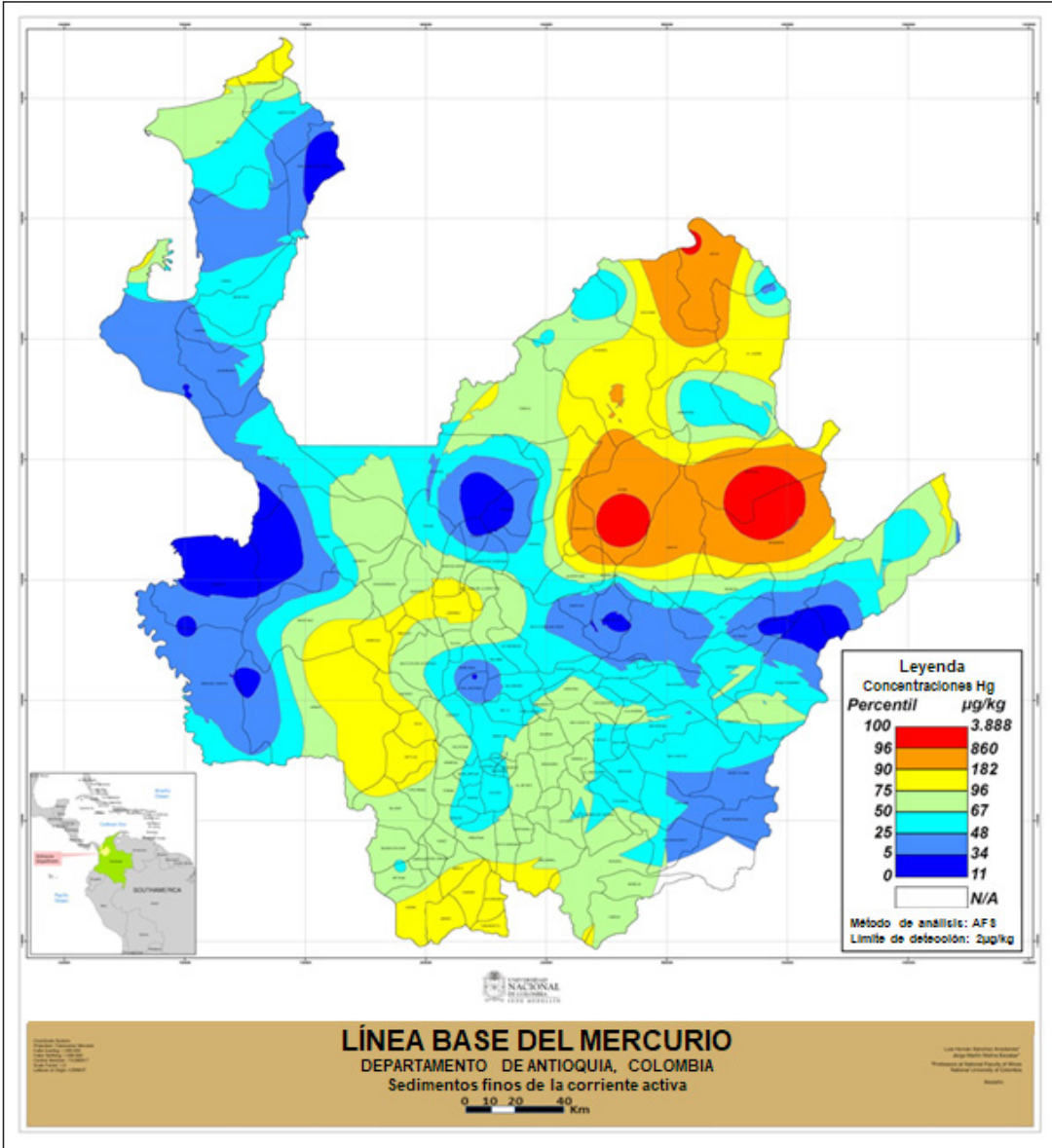

Figura 4. Mapa línea base del mercurio, departamento de Antioquia Fuente: elaboración propia. 


\section{ANÁLISIS DE RESULTADOS}

Los valores bajos de mercurio corresponden a menos del $5 \%$ de los datos estimados $\left(\mathrm{Hg}<34 \mu \mathrm{g} \mathrm{kg}^{-1}\right)$ y cubren varias zonas de la región occidente, Urabá y pequeñas zonas de la región centro y oriente antioqueño.

Los valores altos de $\mathrm{Hg}\left(>182 \mu \mathrm{g} \mathrm{kg}^{-1}\right)$ se encuentran en el nordeste antioqueño relacionados con depósitos de oro y plata, principalmente con las mineralizaciones filonianas de Segovia-Remedios, de Anorí y bajo Cauca, donde se detectaron valores por encima de $860 \mu \mathrm{g} \mathrm{kg}^{-1}$. Estos valores son muy superiores a límite permisible máximo de $150 \mu \mathrm{g} \mathrm{kg}^{-1}$ [1].

Los valores de mercurio entre 67 y $182 \mu \mathrm{g} \mathrm{kg}^{-1}$, representan el $40 \%$ de los valores estimados y pueden estar relacionados con filones epitermales encajados en diferentes tipos de roca, especialmente graníticas, en los municipios de Frontino, Buriticá, algunos municipios del oriente y suroeste antioqueño. La zona norte del Urabá antioqueño y el municipio de Yondó, contienen valores de mercurio entre 67 y $182 \mu \mathrm{g} \mathrm{kg}^{-1}$ los cuales son normales en las litologías drenadas de la zona, siendo probable que exista alguna influencia de los volcanes de lodo, en el caso de la región de Urabá. Esta zona tiene concentraciones levemente superiores al límite permisible.

El mercurio tiene buena correlación con el $\mathrm{Bi}(>0,4)$, el cual hace parte de una de las asociaciones estadísticas más importantes del Hg en el mapa geoquímico de Antioquia de sedimentos activos, en compañía del $\mathrm{Au}, \mathrm{Cu}, \mathrm{Cr}, \mathrm{Mn}, \mathrm{Mo}, \mathrm{Pb}$ y $\mathrm{Zn}$.

\section{CONCLUSIONES}

Se logró establecer la línea base del mercurio para el departamento de Antioquia, que servirá de soporte para nuevos estudios que se realicen, ya sea como elemento guía o para investigaciones de tipo ambiental, que permitan regular su uso en las actividades mineras y entender sus distintos tipos de origen, natural y antrópico.

Las altas concentraciones encontradas en el nordeste antioqueño son un reflejo del alto uso del mercurio para el proceso de amalgamación del oro y de la plata. Esta zona requiere un estricto control por parte de las autoridades ambientales para anular la utilización del mercurio y capacitar a los mineros en nuevas técnicas de recuperación de estos metales. También debe revisarse el origen natural propio de depósitos hidrotermales.

A partir de este artículo se puede evidenciar la necesidad de un estudio acerca de la bioacumulación del mercurio en las especies animales y plantas en el nordeste antioqueño, de su fraccionamiento en distintas especies, su movilidad y su relación con 
lo hidrología, y distinguir las diferentes fuentes del Hg presentes en el sistema, para saber qué tanto puede haber afectado a los habitantes de estas zonas y a los ecosistemas.

\section{AGRADECIMIENTOS}

Esta línea base ha sido elaborada gracias al apoyo económico de la Secretaría de Productividad y Competitividad de la Gobernación de Antioquia y de la Dirección de Investigación (DIME) de la Universidad Nacional de Colombia. El Ingeominas brindó apoyo logístico, con especial agradecimiento en esta institución al geólogo Gabriel Salazar quien levantó la base de datos histórica. Los estudiantes de la Facultad de Minas Kevin A. Van Grieten, León J. Congote, Eliu Pérez, Felipe Flórez, Andrés Mauricio Lara, Gisela Contreras, Oriana Gómez y María Elena Vargas acompañaron la toma de muestras y la preparación para su análisis geoquímico, y agradecemos a Marvin Mosquera, Marcelo del Coral y María Alejandra Campos por su ayuda en la edición final.

\section{REFERENCIAS}

[1] A. Kabata-Pendias y H. Pendias, Trace elements in soils and plants. Florida: CRC press LLC, 2001.

[2] R. Salminen (Editor), Geochemical Atlas of Europe: Part 1- Background information. Finlandia: Geological survey of finland, 2005.

[3] N. Greenwood y A. Earnshaw, Chemistry of the elements. Oxford: Pergamon press, 1984.

[4] M. Mihaljevic et al., Encyclopedia of chemistry, Dordrecht: Kluwer Academic publishers, 1999.

[5] M. Ravichandran, "Interaction between mercury and dissolved organic matter-a review," Chemosphere, vol. 55, n. ${ }^{\circ}$, pp. 319-331, 2004.

[6] D. Brookins, Eh- pH diagrams for geochemistry, Berlín: Springer-Verlag, 1988.

[7] H. González, Mapa geológico del departamento de Antioquia, Medellín: Ingeominas, 1999.

[8] L. Sánchez y J. Molina, Geochemical map of stream sediments of the Antioquia departmentMgased, Medellín, 2009. 